\title{
La discriminación lingüística en estudiantes de bilingüismo de nativa.
}

Linguistic discrimination in nativa bilingualism students.

Luisa Nataly Aguilar Mescco ${ }^{\mathrm{a}}$, Emma Lourdes Durand Gómez ${ }^{\mathrm{a}}$, Mauricio Vilca Rodríguez

${ }^{a}$ Instituto de Educación Superior Pedagógico Público. Arequipa Perú.

bUniversidad Nacional de San Agustín. Arequipa Perú.

\section{INFORMACIÓN}

Historia del Artículo

Recepción: 07/02/2020

Revisión: 05/05/2020

Aceptación: 20/05/2020

\section{Palabras Clave}

Sociolingüística,

lingüísticas,

Habilidades

Lingüística, Bilingüismo Nativa.

\section{Key Words}

Sociolinguistics, Linguistic skills, Linguistic Discrimination, Native Bilingualism.

\section{DOI}

https://doi.org/10.35286/veritas. v21i2.272

\begin{abstract}
RESUMEN
El trabajo de investigación se desarrolló en el Instituto de Educación Superior Pedagógico Público de Arequipa, con el propósito de analizar, en primer lugar, si existe discriminación lingüística hacia los bilingües de nativa y, en segundo término, conocer cuáles son las formas de tal exclusión. Para la operacionalización de variables se consideró las siguientes dimensiones: bilingüismo de nativa (quechua-castellano; aimara-castellano) y cuatro formas de discriminación lingüística. El tipo de investigación es descriptiva, de diseño ex-post-facto, desarrollada bajo el enfoque cuantitativo. Se utilizó la encuesta y un cuestionario de 21 preguntas para el recojo de información; con estos materiales pudimos obtener datos respecto de las diferentes formas de discriminación lingüística hacia los bilingües nativos quechua-aimaras, dentro de la institución en mención. La población está constituida por el $100 \%$ de los estudiantes del IESPPA. Las conclusiones evidencian la existencia de discriminación lingüística hacia los bilingües de nativa, debido a que la mayoría de ellos aún no tiene claro las variedades lingüísticas y que en nuestro medio persiste el prejuicio de que hay una sola norma estándar, que se presenta como única y válida y esta se debe aplicar en todas las situaciones y contextos comunicativos.
\end{abstract}

\begin{abstract}
The research work was carried out at the Institute of Public Pedagogical Higher Education of Arequipa, with the purpose of analyzing, firstly, if there is linguistic discrimination towards native bilinguals and, secondly, to know what are the forms of such exclusion. For the operationalization of variables, the following dimensions were considered: native bilingualism (Quechua-Castilian; Aymara-Castilian) and four forms of linguistic discrimination. The type of research is descriptive, ex-post-facto design, developed under the quantitative approach. The survey and a 21-question questionnaire were used to collect information; With these materials we were able to obtain data regarding the different forms of linguistic discrimination towards native Quechua-Aymara bilinguals, within the institution in question. The population is made up of $100 \%$ of IESPPA students. The conclusions show the existence of linguistic discrimination towards native bilinguals, because most of them are still unclear about the linguistic varieties and that in our environment the prejudice persists that there is only one standard norm, which is presented as unique and valid and this must be applied in all communicative situations and contexts.
\end{abstract}

\section{INTRODUCCIÓN}

La discriminación lingüística constituye un tema de interés educativo y social, por cuanto está considerado como una forma de racismo vigente en el contexto latinoamericano y mundial. Así, tenemos que para Escobar (1972) en América Latina existe discriminación por parte de algunos hablantes hacia muchos otros hombres o mujeres que hablan una lengua distinta a la oficial, esta forma de maltrato se extiende a organismo estatales, incluso la escuela. Aunque hay pocas investigaciones sobre este tema, día a día observamos que la dificultad para construir enunciados sintácticamente alineados a lo señalado por la Academia de la Lengua Española, genera burlas y discriminación. De manera similar, en el trabajo de Hernández-Rosete y Maya (2015) se

Correspondencia:

Mauricio Vilca Rodríguez

mvilcaro@unsa.edu.pe define la discriminación lingüística escolar también como un problema social poco documentado, pero encontraron que la pobreza, la condición migrante y el trabajo infantil agudizan esta situación. A partir de lo señalado podemos enfatizar la importancia de realizar investigaciones que permitan conocer si en las instituciones educativas de nivel superior en el Perú existe discriminación vinculada al contacto de lenguas y de qué forma se manifiesta; asimismo, resulta de interés social identificar las formas de violencia generadas en contextos bilingües.

A partir de la revisión de estudios empíricos sobre discriminación lingüística hemos logrado recuperar un total de quince artículos publicados en revistas indexadas en bases de datos de acceso abierto como Scielo, Redalyc e incluso Google Académico, de los cuales se seleccionaron los siguientes. El primero, denominado "El Programa de Educación Intercultural Bilingüe y sus resultados: ¿perpetuando la discriminación?, de Lagos, 2015, señala que en el contexto de la educación intercultural bilingüe de Chile se ha identificado la ruptura de los circuitos tradicionales de producción y reproducción de las lenguas 
indígenas, originando la vitalidad de las mismas y generando discriminación, asimismo, se encontró que el currículo educativo no considera los contenidos de lengua y cultura indígena, dejando a la libre elección para alumnos de ascendencia indígena, mas no para todo estudiante chileno. Podemos evidenciar que dicha situación genera actos discriminatorios de carácter lingüístico y cultural en este país. De otro lado, el artículo: "Identidades sociolingüísticas y migración internacional. Reacciones frente a la discriminación", de Avilés e Ibarra (2016), discute las formas en que dos comunidades indígenas mexicanas enfrentan la discriminación sociolingüística en la era de la globalización, a partir de la experiencia de los migrantes originarios de Santa Catarina y de los migrantes mixtecos de San Juan Mixtepec, en el contexto nacional estadounidense; se evidencia que la globalización es un fenómeno de intensificación comunicativa que puede, ciertamente, diseminar ideologías de superioridad lingüística y, por extensión un imperialismo lingüístico y cultural.

Los trabajos de investigación hasta aquí analizados contribuyen a reforzar la idea de la existencia de situaciones reales de discriminación lingüística en el contexto de los países de nuestra región. El estudio: "Los derechos lingüísticos de los pueblos indígenas del Ecuador: ¿Interculturalidad o asimilación? (2007-2014)”, de Rodríguez (2016), concluye que la gravedad en la que se encuentran las lenguas indígenas y el derecho a la educación intercultural bilingüe es evidente. En el mismo sentido de analizar la discriminación por factores lingüísticos motivó el trabajo: “Actitudes lingüísticas en contextos interculturales: población asháninka Bajo Chirani” de Falcón y Mamani (2017), concluye que una de las actitudes lingüísticas referida a la conciencia lingüística de la población bilingüe Asháninka hace posible que diferencien e identifiquen entre indígenas y no indígenas con las connotaciones negativas encubiertas para los indígenas. Cabe puntualizar que la población encuestada sí puso de manifiesto su preferencia por la lengua castellana sobre la lengua asháninka, asignando características más positivas a la primera tanto en el aspecto social y personal. Los resultados de esta investigación demuestran una vez más que en condiciones de bilingüismo se generan situaciones de intolerancia por parte de los hablantes que otorgan mayor valor a una lengua respecto de las lenguas originarias.

Por tales consideraciones, el presente trabajo de investigación busca identificar la presencia de conductas orientadas a la discriminación lingüística de nativa y a la vez la prevalencia de algunas formas discriminatorias evidenciadas a través de actitudes excluyentes, sarcasmo, burlas y expresiones kinésicas a partir de situaciones comunicativas reales donde los informantes tengan la oportunidad de mostrar diversas habilidades lingüísticas de comprensión y producción oral y escrita. La investigación se ejecutó en el Instituto de Educación Superior Pedagógico Público Arequipa, tomando como población al $100 \%$ de estudiantes, es decir, se realizó el estudio con los alumnos de siete especialidades, estas son: Comunicación, Educación Básica Alternativa, Educación Inicial, Educación Física, Educación Primaria, Idiomas y Matemática. Para el recojo de información se aplicó un cuestionario que permitió visualizar que existe una importante cantidad de bilingües que tienen como primera lengua el quechua $y$, en menor porcentaje el aimara. Como hipótesis de este estudio se plantea que existe discriminación lingüística en los hablantes Quechua Castellano, Aimara - Castellano.

\section{MATERIAL Y MÉTODOS}

El tipo de investigación es descriptivo de diseño ex -postfacto y se desarrolla bajo el enfoque cuantitativo. El método es hipotético- deductivo porque a partir de supuestos teóricos analizamos un hecho lingüístico y deducimos los resultados por los datos obtenidos en el proceso de investigación (Sampieri, 2018). La población está conformada por el total de estudiantes del Instituto (410 estudiantes), con la finalidad de obtener resultados válidos y evitar cualquier tipo de sesgo en la confirmación de la hipótesis de la investigación. La recolección de datos se realizó empleando la técnica de la encuesta, que permitió recabar información, a través de un cuestionario diseñado con 21 ítems y validado con cinco expertos en la materia. Previo consentimiento informado, se inició la etapa de recolección de datos con la presentación del equipo de investigación a los estudiantes de cada uno de los semestres, según la especialidad: Comunicación, Educación Básica Alternativa, Educación Inicial, Educación Física, Educación Primaria, Idiomas y Matemática. Seguidamente se explica que el propósito de la encuesta busca obtener información respecto de las variables de investigación, por ello se les solicitó el apoyo correspondiente. Para el procesamiento de la información se utilizó la estadística descriptiva con apoyo del programa informático Excel.

\section{RESULTADOS}

Los estudiantes monolingües del Instituto de Educación Superior Pedagógico Público Arequipa manifiestan actitudes y comportamientos asociados directamente con la Discriminación Lingüística contra los bilingües de nativa, debido a que la mayoría de ellos aún no es consciente de la existencia de variedades lingüísticas a nivel de uso del español en condiciones de contacto de lenguas: Quechua Castellano y Aimara- Castellano; considerando erróneamente que en nuestro medio hay una sola norma estándar que se presenta como única y válida y esta se debe aplicar en todas las situaciones y contextos comunicativos generando algún tipo de violencia psicológica por el uso distinto de la lengua española.

\section{Tabla 1: Comprensión Escrita}

\begin{tabular}{|c|c|c|c|c|c|c|c|c|c|c|}
\hline \multirow{2}{*}{ Especialidad } & \multicolumn{2}{|c|}{ No sabe } & \multicolumn{2}{|c|}{ Básico } & \multicolumn{2}{|c|}{ Intermedio } & \multicolumn{2}{|c|}{ Avanzado } & \multicolumn{2}{|c|}{ Total } \\
\hline & $\mathrm{F}$ & $\%$ & F & $\%$ & $\mathrm{~F}$ & $\%$ & F & $\%$ & $\mathrm{~F}$ & $\%$ \\
\hline Comunicación & 12 & $26 \%$ & 8 & $17 \%$ & 9 & $19 \%$ & 18 & $38 \%$ & 47 & \\
\hline $\begin{array}{l}\text { Educación Bási- } \\
\text { ca Alternativa }\end{array}$ & 3 & $14 \%$ & 4 & $18 \%$ & 6 & $27 \%$ & 9 & $41 \%$ & 22 & \\
\hline $\begin{array}{c}\text { Educación } \\
\text { Inicial }\end{array}$ & 20 & $14 \%$ & 38 & $27 \%$ & 37 & $26 \%$ & 45 & $32 \%$ & 140 & \\
\hline $\begin{array}{l}\text { Educación } \\
\text { Física }\end{array}$ & 40 & $47 \%$ & 16 & $19 \%$ & 9 & $11 \%$ & 20 & $24 \%$ & 85 & \\
\hline $\begin{array}{c}\text { Educación } \\
\text { Primaria }\end{array}$ & 8 & $31 \%$ & 5 & $19 \%$ & 3 & $12 \%$ & 10 & $38 \%$ & 26 & \\
\hline Idioma & 24 & $40 \%$ & 15 & $25 \%$ & 9 & $15 \%$ & 12 & $20 \%$ & 60 & \\
\hline Matemática & 7 & $23 \%$ & 8 & $27 \%$ & 3 & $10 \%$ & 12 & $40 \%$ & 30 & \\
\hline TOTAL & & & & & & & & & 410 & $100 \%$ \\
\hline
\end{tabular}




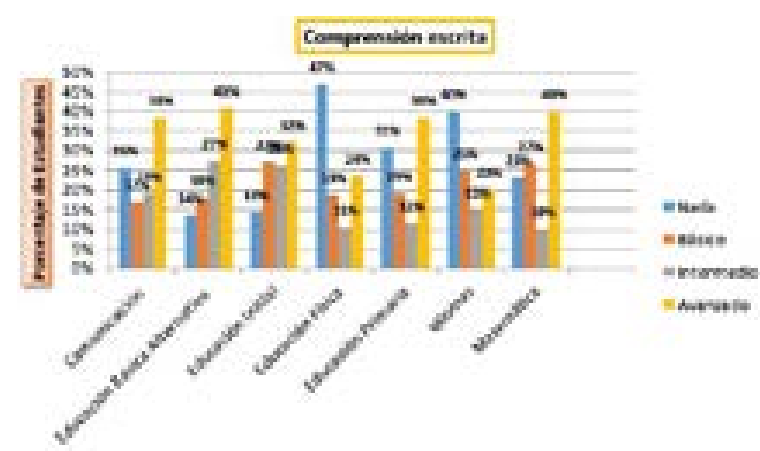

Los resultados del gráfico anterior abordan las habilidades lingüísticas que fueron analizadas para determinar el grado de empleo de un dialecto del español por parte de los bilingües de nativa en el ISPA. En este cuadro se muestra que los estudiantes bilingües Quechua-Castellano desarrollan la comprensión de textos escritos en L1 cuando lo vinculan con un contexto en particular y esto conlleva al lector a involucrarse en una serie de procesos inferenciales necesarios para ir construyendo significados a medida que realizan la lectura.

\section{Tabla 2: Comprensión Oral}

\begin{tabular}{|c|c|c|c|c|c|c|c|c|c|c|}
\hline \multirow{2}{*}{ Especialidad } & \multicolumn{2}{|c|}{ No sabe } & \multicolumn{2}{|c|}{ Básico } & \multicolumn{2}{|c|}{ Intermedio } & \multicolumn{2}{|c|}{ Avanzado } & \multicolumn{2}{|c|}{ Total } \\
\hline & $\mathrm{F}$ & $\%$ & $\mathrm{~F}$ & $\%$ & $\mathrm{~F}$ & $\%$ & $\mathrm{~F}$ & $\%$ & F & $\%$ \\
\hline Comunicación & 15 & $32 \%$ & 5 & $11 \%$ & 11 & $23 \%$ & 16 & $34 \%$ & 47 & \\
\hline $\begin{array}{l}\text { Educación Básica } \\
\text { Alternativa }\end{array}$ & 2 & $9 \%$ & 7 & $32 \%$ & 5 & $23 \%$ & 8 & $36 \%$ & 22 & \\
\hline Educación Inicial & 16 & $11 \%$ & 15 & $11 \%$ & 48 & $34 \%$ & 61 & $44 \%$ & 140 & \\
\hline Educación Física & 44 & $52 \%$ & 12 & $14 \%$ & 10 & $12 \%$ & 19 & $22 \%$ & 85 & \\
\hline $\begin{array}{c}\text { Educación } \\
\text { Primaria }\end{array}$ & 10 & $38 \%$ & 5 & $19 \%$ & 2 & $8 \%$ & 9 & $35 \%$ & 26 & \\
\hline Idioma & 29 & $48 \%$ & 15 & $25 \%$ & 5 & $8 \%$ & 11 & $18 \%$ & 60 & \\
\hline Matemática & 9 & $30 \%$ & 5 & $17 \%$ & 5 & $17 \%$ & 11 & $37 \%$ & 30 & \\
\hline Total & & & & & & & & & 410 & $100 \%$ \\
\hline
\end{tabular}

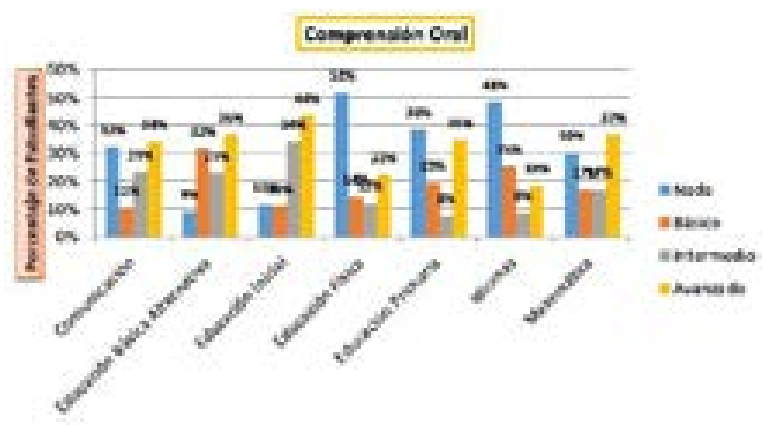

En relación a la competencia de comprensión oral existen un porcentaje considerable de estudiantes quechuahablantes del Pedagógico Arequipa que comprenden textos orales en diferentes situaciones y contextos comunicativos, esto nos permite deducir que identifican información básica y varios detalles dispersos en un texto oral con temática variada.
Tabla 3: Producción Escrita

\begin{tabular}{ccccccccccc}
\hline \multirow{2}{*}{ Especialidad } & \multicolumn{2}{c}{ No sabe } & \multicolumn{2}{c}{ Básico } & \multicolumn{2}{c}{ Intermedio } & \multicolumn{2}{c}{ Avanzado } & \multicolumn{2}{c}{ Total } \\
\cline { 2 - 11 } & $\mathrm{F}$ & $\%$ & $\mathrm{~F}$ & $\%$ & $\mathrm{~F}$ & $\%$ & $\mathrm{~F}$ & $\%$ & $\mathrm{~F}$ & $\%$ \\
\hline Comunicación & 32 & $68 \%$ & 11 & $23 \%$ & 1 & $2 \%$ & 3 & $6 \%$ & 47 & \\
$\begin{array}{c}\text { Educación Básica } \\
\text { Alternativa }\end{array}$ & 10 & $45 \%$ & 10 & $45 \%$ & 2 & $9 \%$ & 0 & $0 \%$ & 22 & \\
Educación Inicial & 43 & $31 \%$ & 74 & $53 \%$ & 17 & $12 \%$ & 6 & $4 \%$ & 140 & \\
Educación Física & 65 & $76 \%$ & 4 & $5 \%$ & 8 & $9 \%$ & 8 & $9 \%$ & 85 & \\
Educación & 16 & $62 \%$ & 5 & $19 \%$ & 2 & $8 \%$ & 3 & $12 \%$ & 26 & \\
Primaria & & & & & & & & & & \\
Idioma & 45 & $75 \%$ & 12 & $20 \%$ & 2 & $3 \%$ & 1 & $2 \%$ & 60 & \\
Matemática & 21 & $70 \%$ & 8 & $27 \%$ & 1 & $3 \%$ & 0 & $0 \%$ & 30 & \\
\hline Total & & & & & & & & 410 & $100 \%$ \\
\hline
\end{tabular}

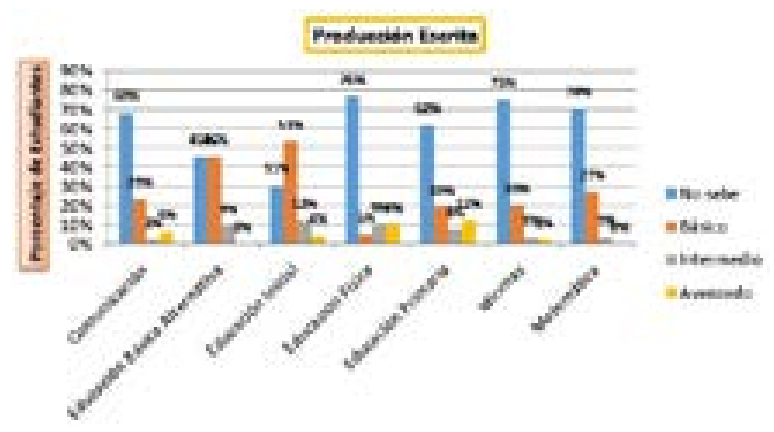

La competencia de producción escrita muestra un gran porcentaje de bilingües que no saben escribir en quechua; en otras palabras, son pocos los estudiantes que tienen un dominio en la producción de textos escritos, esto nos ratifica nuevamente que aún no se escribe en lengua nativa y que los mismos hablantes de quechua admiten no textualizar experiencias, ideas y sentimientos empleando las convenciones del lenguaje escrito.

\section{Tabla 4: Expresión Oral}

\begin{tabular}{ccccccccccc}
\hline \multirow{2}{*}{ Especialidad } & \multicolumn{2}{c}{ No sabe } & \multicolumn{2}{c}{ Básico } & \multicolumn{2}{c}{ Intermedio } & \multicolumn{2}{c}{ Avanzado } & \multicolumn{2}{c}{ Total } \\
\cline { 2 - 10 } & $\mathrm{F}$ & $\%$ & $\mathrm{~F}$ & $\%$ & $\mathrm{~F}$ & $\%$ & $\mathrm{~F}$ & $\%$ & $\mathrm{~F}$ & $\%$ \\
\hline Comunicación & 29 & $62 \%$ & 5 & $11 \%$ & 4 & $9 \%$ & 9 & $19 \%$ & 47 & \\
$\begin{array}{c}\text { Educación Básica } \\
\text { Alternativa }\end{array}$ & 5 & $23 \%$ & 6 & $27 \%$ & 4 & $18 \%$ & 7 & $32 \%$ & 22 & \\
Educación inicial & 18 & $13 \%$ & 24 & $17 \%$ & 42 & $30 \%$ & 56 & $40 \%$ & 140 & \\
$\begin{array}{c}\text { Educación física } \\
\text { Educación }\end{array}$ & 35 & $41 \%$ & 21 & $25 \%$ & 7 & $8 \%$ & 22 & $26 \%$ & 85 & \\
primaria & 9 & $35 \%$ & 3 & $12 \%$ & 1 & $4 \%$ & 13 & $50 \%$ & 26 & \\
Idioma & 32 & $53 \%$ & 10 & $17 \%$ & 7 & $12 \%$ & 11 & $18 \%$ & 60 & \\
Matemática & 9 & $30 \%$ & 5 & $17 \%$ & 4 & $13 \%$ & 12 & $40 \%$ & 30 & \\
\hline Total & & & & & & & & 410 & $100 \%$ \\
\hline
\end{tabular}




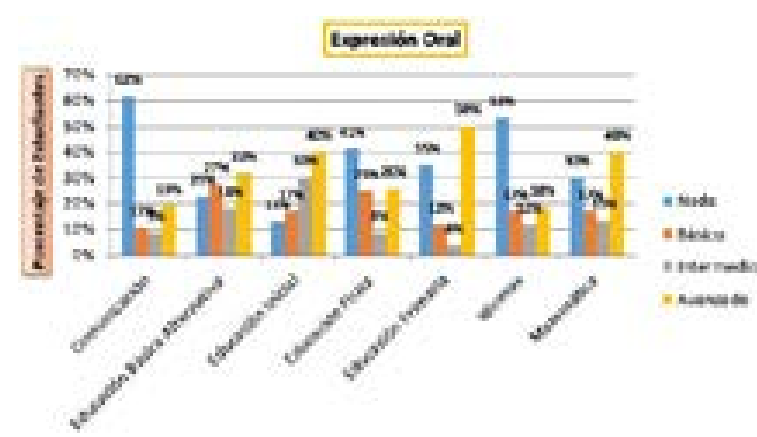

En el gráfico anterior, se visualiza que un notable porcentaje de estudiantes desarrollan la expresión oral en quechua; en otros términos, ordenan sus ideas en torno a un tema específico a partir de sus saberes previos y fuentes de información, evitando vacíos de información.

Tabla 5: ¿Qué opina usted de esta forma de emplear el castellano?

\begin{tabular}{|c|c|c|c|c|c|c|c|c|c|c|}
\hline \multirow{2}{*}{ Especialidad } & \multicolumn{2}{|c|}{ A } & \multicolumn{2}{|c|}{ B } & \multicolumn{2}{|c|}{$\mathrm{C}$} & \multicolumn{2}{|c|}{ D } & \multicolumn{2}{|c|}{ Total } \\
\hline & F & $\%$ & F & $\%$ & F & $\%$ & F & $\%$ & F & $\%$ \\
\hline Comunicación & 9 & $19 \%$ & 18 & $38 \%$ & 7 & $15 \%$ & 13 & $28 \%$ & 47 & \\
\hline $\begin{array}{c}\text { Educación Básica } \\
\text { Alternativa }\end{array}$ & 10 & $45 \%$ & 9 & $41 \%$ & 3 & $14 \%$ & 0 & $0 \%$ & 22 & \\
\hline Educación Inicial & 15 & $11 \%$ & 73 & $52 \%$ & 49 & $35 \%$ & 3 & $2 \%$ & 140 & \\
\hline Educación Física & 27 & $32 \%$ & 30 & $35 \%$ & 12 & $14 \%$ & 16 & $19 \%$ & 85 & \\
\hline $\begin{array}{c}\text { Educación } \\
\text { Primaria }\end{array}$ & 10 & $38 \%$ & 14 & $54 \%$ & 2 & $8 \%$ & 0 & $0 \%$ & 26 & \\
\hline Idioma & 26 & $43 \%$ & 15 & $25 \%$ & 13 & $22 \%$ & 6 & $10 \%$ & 60 & \\
\hline Matemática & 14 & $47 \%$ & 13 & $43 \%$ & 3 & $10 \%$ & 0 & $0 \%$ & 30 & \\
\hline Promedio \% & & $34 \%$ & & $41 \%$ & & $17 \%$ & & $8 \%$ & & \\
\hline Total & & & & & & & & & 410 & $100 \%$ \\
\hline
\end{tabular}

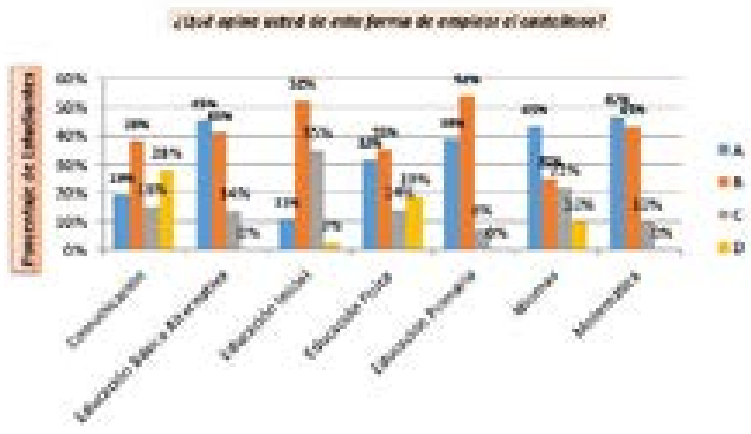

A partir de los datos que se muestran en la figura anterior podemos señalar que la mayoría de los estudiantes del IESPPA ratifican que los bilingües de nativa emplean expresiones consideradas por los informantes como "mala o incorrectas"; este pensamiento es un tipo de discriminación lingüística, pues en la mente de nuestros informantes persiste el hecho de que hay formas "correctas e incorrectas" y que son los nativos quienes expresan términos que no deben ser aceptados en ninguna situación comunicativa. Esta arbitrariedad no contribuye a desarrollar las competencias comunicativas que se pretende incrementar hoy en día en nuestros estudiantes de todos los niveles. Es en la EBR en donde la interculturalidad es una necesidad y ésta se extiende a la educación superior.

Tabla 6: Cuando un quechuahablante/aimarahablante se dirige a diferentes entidades como: poder judicial, servicios públicos, oficinas de la municipalidad, asociación de vecinos, medios de transporte, hospitales, etc.

\begin{tabular}{ccccccccccc}
\hline \multirow{2}{*}{ Especialidad } & \multicolumn{2}{c}{$\mathrm{A}$} & \multicolumn{2}{c}{$\mathrm{B}$} & \multicolumn{2}{c}{$\mathrm{C}$} & \multicolumn{2}{c}{$\mathrm{D}$} & \multicolumn{2}{c}{ Total } \\
\cline { 2 - 9 } & $\mathrm{F}$ & $\%$ & $\mathrm{~F}$ & $\%$ & $\mathrm{~F}$ & $\%$ & $\mathrm{~F}$ & $\%$ & $\mathrm{~F}$ & $\%$ \\
\hline Comunicación & 0 & $0 \%$ & 20 & $43 \%$ & 25 & $53 \%$ & 2 & $4 \%$ & 47 & \\
$\begin{array}{c}\text { Educación Básica } \\
\text { Alternativa }\end{array}$ & 0 & $0 \%$ & 8 & $36 \%$ & 14 & $64 \%$ & 0 & $0 \%$ & 22 & \\
& & & & & & & & & & \\
Educación Inicial & 3 & $2 \%$ & 50 & $36 \%$ & 85 & $61 \%$ & 2 & $1 \%$ & 140 & \\
& & & & & & & & & & \\
Educación Física & 0 & $0 \%$ & 40 & $47 \%$ & 45 & $53 \%$ & 0 & $0 \%$ & 85 & \\
Educación & 0 & $0 \%$ & 8 & $31 \%$ & 18 & $69 \%$ & 0 & $0 \%$ & 26 & \\
Primaria & & & & & & & & & & \\
Idioma & 0 & $0 \%$ & 24 & $40 \%$ & 36 & $60 \%$ & 0 & $0 \%$ & 60 & \\
Matemática & 0 & $0 \%$ & 7 & $23 \%$ & 23 & $77 \%$ & 0 & $0 \%$ & 30 & \\
Promedio \% & & $0 \%$ & & $37 \%$ & & $62 \%$ & & $1 \%$ & & \\
\hline Total & & & & & & & & & 410 & $100 \%$ \\
\hline
\end{tabular}

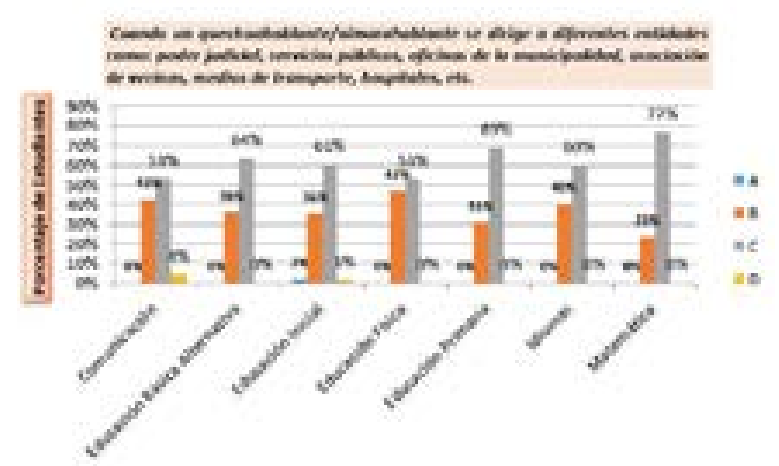

Otra de las formas de Discriminación Lingüística que se consideró a través de la aplicación del cuestionario y la observación fue las actitudes excluyentes, tal como se muestra en la figura anterior. El análisis permite concluir que el $62 \%$ del estudiantado del Pedagógico registraron que el trato hacia los bilingües Q-C es malo; este juicio se entiende debido a que la mayoría de los alumnos de la institución investigada piensan que existen diferencias en las expresiones orales; y éstas conllevan a afianzar los prejuicios en las instituciones superiores y además se le ha asociado a la discriminación lingüística. 
Tabla 7: En el IESPPA, cuando un estudiante tiene dificultades para comunicarse, qué gestos observa usted en los interlocutores.

\begin{tabular}{ccccccccccc}
\hline \multirow{2}{*}{ Especialidad } & \multicolumn{2}{c}{$\mathrm{A}$} & \multicolumn{2}{c}{$\mathrm{B}$} & \multicolumn{2}{c}{$\mathrm{C}$} & \multicolumn{2}{c}{$\mathrm{D}$} & \multicolumn{2}{c}{ Total } \\
\cline { 2 - 9 } & $\mathrm{F}$ & $\%$ & $\mathrm{~F}$ & $\%$ & $\mathrm{~F}$ & $\%$ & $\mathrm{~F}$ & $\%$ & $\mathrm{~F}$ & $\%$ \\
\hline Comunicación & 0 & $0 \%$ & 23 & $49 \%$ & 5 & $11 \%$ & 19 & $40 \%$ & 47 & \\
$\begin{array}{c}\text { Educación Básica } \\
\text { Alternativa }\end{array}$ & 0 & $0 \%$ & 14 & $64 \%$ & 0 & $0 \%$ & 8 & $36 \%$ & 22 & \\
Educación Inicial & 7 & $5 \%$ & 72 & $51 \%$ & 10 & $7 \%$ & 51 & $36 \%$ & 140 & \\
Educación Física & 16 & $19 \%$ & 35 & $41 \%$ & 10 & $12 \%$ & 24 & $28 \%$ & 85 & \\
Educación & 2 & $8 \%$ & 12 & $46 \%$ & 1 & $4 \%$ & 11 & $42 \%$ & 26 & \\
Primaria & & & & & & & & & & \\
Idioma & 4 & $7 \%$ & 17 & $28 \%$ & 19 & $32 \%$ & 20 & $33 \%$ & 60 & \\
Matemática & 1 & $3 \%$ & 13 & $43 \%$ & 5 & $17 \%$ & 11 & $37 \%$ & 30 & \\
Promedio \% & $6 \%$ & & $46 \%$ & & $12 \%$ & & $36 \%$ & & \\
\hline Total & & & & & & & & & 410 & $100 \%$ \\
\hline
\end{tabular}

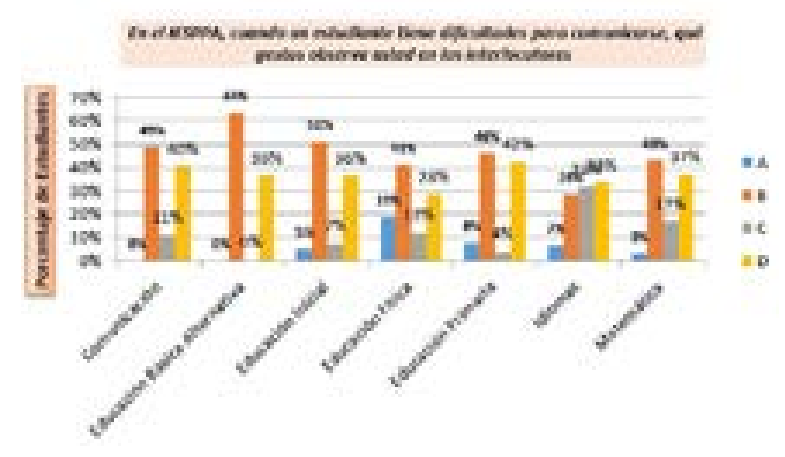

Finalmente, los datos de esta figura revelan distintas formas de discriminación lingüística en relación a expresiones kinésicas, donde el 36\% del total de los estudiantes del Instituto ratifica que la mayoría de los alumnos emplean gestos de discriminación por la forma de hablar, ya que "hablar como serrano" en el Perú significa "emplear de manera inadecuada el lenguaje". Esto se debe a que usamos el lenguaje para crear jerarquías sociales basadas en el uso de la lengua. La norma más ligada con las clases altas se convierte en un nivel para medir a los demás; una mejor ortografía, una sintaxis, etc. Usamos todo eso como una forma de diferencia y separación.

\section{DISCUSIÓN}

Para Ana María Escobar (1988), existen similitudes entre hablantes que adquieren una lengua como primera (bilingüismo de nativa) y los que adquieren una lengua como segunda, sientan las bases para realizar estudios de carácter lingüístico y sociolingüístico, independientemente de las características gramático- estructurales de la primera y segunda lenguas de los hablantes. Las características lingüísticas de los estudiantes del pedagógico público Arequipa corresponden a las categorías: monolingües (hablantes solo de Castellano) y bilingües de nativa (Quechua - Castellano, Aimara - Castellano).
Los resultados de la investigación sobre LA DISCRIMINACIÓN LINGÜÍSTICA EN LOS ESTUDIANTES DE BILNGÜISMO DE NATIVA permiten constatar la existencia de hablantes bilingües Quechua Castellano, Aimara - Castellano, pues utilizan dos lenguas para resolver situaciones de comunicación tanto formales como informales, de modo que esta situación de contacto genera dos tipos de modificación: a nivel oral y en la escritura; en ambos casos se adoptan categorías ajenas a la propia lengua y, propias a la ajena; en este proceso de intercambio de elementos lingüísticos, una de las lenguas tiende a ser considerada inferior por la idea de adoptar lo socialmente superior y prestigioso. Estas condiciones generan diversas manifestaciones de discriminación lingüística como el hecho de creer que algunas expresiones verbales son "malas e incorrectas". Se ha intentado distinguir y definir los factores sociales, culturales y lingüísticos que afectan el comportamiento verbal de la población que forma parte de la investigación.

Corder (1975), plantea que los factores no lingüísticos que intervienen en la discriminación lingüística y que pueden tener consecuencias en el comportamiento verbal bilingüe han sido descritos desde tres perspectivas psicológica, social y lingüística. Este fundamento teórico permite entender el porqué del trato discriminatorio hacia los bilingües de nativa cuando asisten a diversas instituciones tanto públicas como privadas para realización de trámites diversos. También se ha logrado evidenciar que la discriminación lingüística se manifiesta no solo a través de expresiones violentas como "serrano", "cholo", "indio", etc., sino que la presencia de gestos y expresiones racistas y despectivas tienen alta presencia en el Instituto de Educación Superior Pedagógico Público Arequipa.

\section{REFERENCIAS BIBLIOGRÁFICAS}

1. Avilés González, K., \& Ibarra Templos, Y. (2016). Identidades sociolingüísticas y migración internacional. Reacciones frente a la discriminación. Alteridades, 26(51), 73-84. Recuperado de http://www.scielo. org.mx/scielo.php?script=sci_arttext\&pid=S018870172016000100073\&lng=es\&tlng=es.

2. Corder, S.P. (1975). Análisis de errores, interlenguaje $\mathrm{y}$ adquisición de un segundo idioma. Enseñanza de idiomas, 8 (4), 201-218.

3. Escobar, A. (1972). Lenguaje y discriminación social en América Latina. Lima: Milla Batres.

4. Escobar, A. M. (1988). Hacia una tipología del bilingüismo en el Perú. Documento de trabajo No. 28. Lima: IEP Instituto de Estudios Peruanos

5. Falcón, P. \& Mamani Quispe, L. A. (2017). Actitudes lingüísticas en contextos interculturales: población asháninka Bajo Chirani. RLA. Revista de lingüística teórica y aplicada, 55(1), 95-115. En https://dx.doi. org/10.4067/S0718-48832017000100095

6. Hernández-Rosete, D., \& Maya, O. (2016). Discriminación lingüística y contracultura escolar indígena en la Ciudad de México. Revista Latinoamericana de Ciencias Sociales, Niñez y Juventud, 14(2), 1161-1176.

7. Lagos, C. (2015). El Programa de Educación Intercultural Bilingüe y sus resultados: ¿perpetuando la 
discriminación? The Intercultural Bilingual Education Program and its results: Perpetuating discrimination? Pensamiento Educativo. Revista de Investigación Educacional Latinoamericana, 52(1), 84-94.

8. Rodríguez Caguana, A. (2016). Los derechos lingüísticos de los pueblos indígenas del Ecuador: ¿Interculturalidad o asimilación? (2007-2014). Ecuador Debate 98. En https://repositorio.flacsoandes.edu.ec/ bitstream/10469/12168/1/REXTN-ED98-04-Martinez. pdf

9. Sampieri, R. H. (2018). Metodología de la investigación: las rutas cuantitativa, cualitativa y mixta. México: McGraw Hill. 